\title{
Ambulatory blood pressure in children with obstructive sleep apnoea: a community based study
}

\author{
A M Li, ${ }^{1}$ C T Au, ${ }^{1}$ R Y T Sung, ${ }^{1}$ C Ho, ${ }^{2}$ P C Ng, ${ }^{1}$ T F Fok, ${ }^{1}$ Y K Wing ${ }^{2}$
}

- Additional tables are available in a supplement published online only at http://thorax.bmj.com/ content/vol63/issue9

${ }^{1}$ Department of Paediatrics, Prince of Wales and Shatin Hospital, The Chinese University of Hong Kong, Shatin, Hong Kong; ${ }^{2}$ Department of Psychiatry, Prince of Wales and Shatin Hospital, The Chinese University of Hong Kong, Shatin, Hong Kong

Correspondence to:

Dr A M Li, Department of

Paediatrics, Prince of Wales

Hospital, The Chinese University

of Hong Kong, Shatin, Hong

Kong; albertmli@cuhk.edu.hk

Received 24 September 2007 Accepted 7 February 2008

Published Online First 3 April 2008

\section{ABSTRACT}

Background: Childhood obstructive sleep apnoea (OSA) is increasingly being recognised. Its effects on blood pressure (BP) elevation and hypertension are still controversial.

Objective: To evaluate the association between OSA and ambulatory BP in children.

Methods: Children aged 6-13 years from randomly selected schools were invited to undergo overnight sleep study and ambulatory BP monitoring after completing a validated OSA questionnaire. OSA was diagnosed if the obstructive apnoea-hypopnoea index (AHI) was $>1$, and normal controls had $\mathrm{AHI}<1$ and snoring $<3$ nights per week. Children with OSA were subdivided into a mild group (AHI 1-5) and moderate to severe group (AHI $>5$ ). Results: 306 subjects had valid sleep and daytime BP data. Children with OSA had significantly higher BP than normal healthy children during both sleep and wakefulness. BP levels increased with the severity of OSA, and children with moderate to severe disease (AHI $>5$ ) were at significantly higher risk for nocturnal systolic (OR 3.9 (95\% Cl 1.4 to 10.5)) and diastolic (OR 3.3 (95\% Cl 1.4 to 8.1)) hypertension. Multiple linear regression revealed a significant association between oxygen desaturation index and $\mathrm{AHI}$ with daytime and nocturnal $\mathrm{BP}$, respectively, independent of obesity.

Conclusions: OSA was associated with elevated daytime and nocturnal BP, and is an independent predictor of nocturnal hypertension. This has important clinical implications as childhood elevated BP predicts future cardiovascular risks. Future studies should examine the effect of therapy for OSA on changes in BP.

In adults, obstructive sleep apnoea (OSA) is an independent risk factor for hypertension and is involved in the initiation and progression of other cardiovascular diseases. ${ }^{1-3}$ However, corresponding data for the paediatric population are scarce, and the few published studies have provided conflicting results. ${ }^{4-9}$ Marcus and colleagues ${ }^{4}$ suggested that children with OSA had higher daytime and nocturnal diastolic blood pressure (BP) compared with primary snorers, whereas Amin and colleagues $^{5}$ found lower diastolic BP among children with OSA. Guilleminault and colleagues ${ }^{6}$ demonstrated that a subgroup of children with OSA were hypotensive rather than hypertensive. Kohyama and colleagues ${ }^{7}$ found a positive correlation between both nocturnal systolic and diastolic BP with severity of OSA and, similarly, Enright and colleagues $^{8}$ found respiratory disturbance index to be significantly associated with systolic and diastolic BP. A recent study, however, failed to confirm such a positive association. ${ }^{9}$ These inconsistent findings in $\mathrm{BP}$ measurements might have been related to the small sample sizes and lack of normal healthy subjects for comparison. A recent meta-analysis concluded that there was inadequate evidence for an increased risk of elevated $\mathrm{BP}$ in children with OSA. The authors also found marked heterogeneity among published series and emphasised the need for further studies to clarify this important issue. ${ }^{10}$

In some of the reported paediatric series, casual "on the spot" BP measurements were used. Ambulatory blood pressure (ABP) measurement is a better diagnostic tool as it gives more reliable and reproducible results. ${ }^{11}$ Obesity is becoming an important factor in the study of OSA. Obesity increases the risk of both OSA and cardiovascular disease for which hypertension is the preceding step. Failing to control for obesity will render assessment of the independent role of OSA on BP difficult.

In this study, we aimed to compare $\mathrm{ABP}$ parameters of children suffering from mild and moderate to severe OSA with normal healthy controls, and to investigate the correlation between severity of OSA and BP measures or hypertension, after adjusting for obesity.

\section{METHODS}

\section{Subjects and study design}

Subjects were aged between 6 and 13 years and were recruited from 13 randomly selected schools. Parents were asked to complete a validated OSA screening questionnaire ${ }^{12}$ that stratified children into high or low risk for OSA. All high risk and a randomly selected sample from the low risk group were invited to undergo overnight polysomnographic study (PSG) and ABP monitoring. Children were excluded if they had an intercurrent illness within 4 weeks of PSG, suffered from cardiac, renal or neuromuscular diseases, or if they had chromosomal abnormalities or had previously undergone upper airway surgery. Children receiving medications that could affect BP measurements were also excluded. Anthropometric parameters, including weight, height, and waist and hip circumferences were measured on the day of PSG. Body mass index (BMI) was converted to BMI z score, according to the normal reference. ${ }^{13}$ Children were defined as overweight or obese if their BMI $\mathrm{z}$ score was $>1.04$ or 1.65 , corresponding to the 85 th or 95 th percentile (relative to age and gender), respectively. The study included the first 466 consecutive children who consented to PSG and ABP recordings.

Subjects were classified into three groups according to the PSG and questionnaire results. 
- Group 1: healthy control group (obstructive apnoeahypopnoea index (AHI) $<1$ and history of snoring $<3$ nights per week).

- Group 2: mild OSA group (AHI 1-5).

- Group 3: moderate to severe OSA (AHI >5).

Subjects with primary snoring (AHI $<1$, history of snoring $>3$ nights per week) were not included in the study.

\section{Polysomnography}

All recruited children underwent a standard overnight PSG at a dedicated sleep laboratory with a CNS 1000P polygraph (CNS, Inc., Chanhassen, Minnesota, USA), as described in our previous publication. ${ }^{14}$ All computerised sleep data were further manually edited by experienced PSG technologists and clinicians according to standard criteria. ${ }^{15}$ For definitions of PSG parameters, please refer to our online supplementary file.

\section{Ambulatory blood pressure measurement}

$\mathrm{ABP}$ was monitored on the same day as overnight PSG using an oscillometric monitor (SpaceLabs 90217; SpaceLabs Medical, Redmond, Washington, USA), which has been validated for use in children. ${ }^{16}$ Subjects arrived at the sleep laboratory at 14:00 on the day of PSG, and ABP recordings began at arrival until the subjects were discharged home the next day. Systolic, diastolic and mean arterial BP were measured hourly during the period starting from 21:30 to 07:00, and every 30 min outside of this period. The correct size cuff was placed on the non-dominant arm. The exact cut-off time dividing wake and sleep BP was defined individually according to the PSG tracings. Recordings were included in the analysis when they had a minimum of seven successful readings during active wakefulness and at least seven successful readings during sleep. ${ }^{16}$ Individual mean systolic, diastolic and mean arterial BP were calculated for wake and sleep periods. All mean BP variables were converted into BP $z$ scores using the "LMS" reference values (relative to gender and height) published by Wuhl and colleagues. ${ }^{17}$ In brief, the authors had derived height and gender specific estimates of the distribution median $(\mathrm{M})$, coefficient of variation $(\mathrm{S})$ and degree of skewness (L), which were estimated by a maximum likelihood of curve fitness technique. ${ }^{18}$ These estimated parameters were used to transform the skewed data to normality, giving a more accurate $\mathrm{ABP} z$ score. Elevated $\mathrm{BP}$ and hypertension were defined as mean BP values $>90$ th and 95 th percentiles of the $\mathrm{ABP}$ reference, respectively. ${ }^{17}$

Nocturnal dipping of systolic BP, diastolic BP and mean arterial pressure were derived by calculating the difference between mean awake BP and mean sleep BP and expressed as a percentage of mean awake BP. Subjects with nocturnal BP dipping less than $10 \%$ were defined as non-dippers. ${ }^{19} 20$

\section{Statistical analysis}

Mean (SD), median (interquartile range) and number (percentage) are presented for parametric, non-parametric and categorical data, respectively. Parametric and non-parametric data were compared using one way analysis of variance (ANOVA) and the Kruskal-Wallis test, respectively. For parametric data, the Tukey or Games-Howell method was used for post hoc pairwise comparisons with adjustments made depending on the agreement of the assumption of variance. For non-parametric data, Mann-Whitney tests with adjusted p values (significant at $\mathrm{p}<0.016$ ) were used for pairwise comparisons. As some of the polysomnographic data were skewed and contained zero or extreme values, these variables were logarithmically transformed (natural $\log [\mathrm{x}+0.1]) . \chi^{2}$ tests were performed to investigate the difference in proportions between groups. Multiple $\chi^{2}$ tests with adjusted $p$ values (significant at $\mathrm{p}<0.016$ ) were used for multiple pairwise comparisons.

Table 1 Demographic, anthropometric and polysomnographic data

\begin{tabular}{|c|c|c|c|c|c|}
\hline & $\begin{array}{l}\text { Entire sample } \\
(\mathrm{n}=306)\end{array}$ & $\begin{array}{l}\text { AHI } \leqslant 1 \\
(\mathrm{n}=127)\end{array}$ & $\begin{array}{l}1<\text { AHI } \leqslant 5 \\
(n=133)\end{array}$ & $\begin{array}{l}\text { AHI }>5 \\
(n=46)\end{array}$ & $\begin{array}{l}\mathrm{p} \text { Value } \\
\text { (trend) }\end{array}$ \\
\hline Age (y) & $10.4(1.7)$ & $10.4(1.7)$ & $10.6(1.6)$ & $10.1(1.6)$ & 0.59 \\
\hline Male gender (n (\%)) & $199(65)$ & $72(57)$ & $92(69)$ & $35(76)$ & 0.008 \\
\hline Body height $(\mathrm{cm})$ & $139.6(10.5)$ & $139.0(10.4)$ & $140.2(10.8)$ & $139.5(10.1)$ & 0.58 \\
\hline Body weight $(\mathrm{kg})^{*} \dagger$ & $36.4(10.6)$ & $34.2(9.4)$ & $37.5(11.5)$ & $39.6(10.3)$ & $<0.001$ \\
\hline Body mass index $\left(\mathrm{m} / \mathrm{kg}^{2}\right)^{*} \dagger$ & $18.4(3.4)$ & $17.4(2.9)$ & $18.7(3.5)$ & $20.1(3.4)$ & $<0.001$ \\
\hline Body mass index z score ${ }^{*} \dagger$ & $0.48(1.04)$ & $0.18(0.97)$ & $0.56(1.04)$ & $1.07(0.94)$ & $<0.001$ \\
\hline Normal weight $(\mathrm{n}(\%))^{*} \dagger \dagger$ & $204(66.7)$ & $103(81.1)$ & $85(63.9)$ & $16(34.8)$ & \\
\hline Overweight $(\mathrm{n}(\%))^{*}+*$ & $59(19.3)$ & $16(12.6)$ & $28(21.1)$ & $15(32.6)$ & $<0.001$ \\
\hline Obese $(\mathrm{n}(\%))^{*}+*$ & $43(14.1)$ & $8(6.3)$ & $20(15.0)$ & $15(32.6)$ & \\
\hline Waist circumference $(\mathrm{cm})^{*} \uparrow$ & $63.7(9.5)$ & $60.9(8.1)$ & $64.7(9.9)$ & $68.7(9.5)$ & $<0.001$ \\
\hline Hip circumference $(\mathrm{cm})^{*} \dagger$ & $74.4(9.5)$ & $72.5(9.0)$ & $75.2(10.0)$ & $77.5(8.3)$ & $<0.001$ \\
\hline Waist to hip ratio* ${ }^{*}+$ & $0.86(0.06)$ & $0.84(0.05)$ & $0.86(0.05)$ & $0.89(0.06)$ & $<0.001$ \\
\hline Epworth sleepiness scale score & $6.5(4.1)$ & $6.2(4.1)$ & $6.6(4.2)$ & $6.8(3.9)$ & 0.32 \\
\hline Rapid eye movement sleep (\%) & $21.0(4.1)$ & $21.0(3.9)$ & $21.0(3.9)$ & $20.8(5.1)$ & 0.78 \\
\hline Slow wave sleep (\%) & $22.8(5.2)$ & $23.1(5.4)$ & $22.4(5.1)$ & $23.2(5.0)$ & 0.80 \\
\hline Non-slow wave sleep $(\%)$ & $56.2(5.9)$ & $55.9(6.0)$ & $56.6(5.6)$ & $56.0(6.5)$ & 0.68 \\
\hline Sleep efficiency (\%) & $82.1(10.7)$ & $82.7(10.0)$ & $81.3(10.9)$ & $82.4(12.2$ & 0.64 \\
\hline Apnoea-hypopnoea index $(/ \mathrm{h})^{*}+$ t & $1.2(0.3-2.9)$ & $0.2(0.0-0.5)$ & $2.0(1.3-2.8)$ & $8.2(6.5-13.1)$ & $<0.001$ \\
\hline Oxygen desaturation index $(/ \mathrm{h})^{*}+\$$ & $0.4(0.1-1.0)$ & $0.1(0.0-0.4)$ & $0.5(0.2-1.0)$ & $3.1(1.1-6.1)$ & $<0.001$ \\
\hline Arousal index $(/ \mathrm{h})^{*}+\dagger$ & $6.8(5.2-8.8)$ & $5.7(4.5-7.5)$ & $7.0(5.6-8.8)$ & $12.2(8.0-16.3)$ & $<0.001$ \\
\hline $\mathrm{SpO}_{2}$ nadir $(\%)^{*} \dagger \dagger$ & $91.5(3.2)$ & $92.4(2.1)$ & $91.6(2.6)$ & $88.9(5.1)$ & $<0.001$ \\
\hline
\end{tabular}

Mean (SD), median (IOR) and number (\%) are presented for parametric, non-parametric and categorical data, respectively. The percentages of normal weight, overweight and obese patients do not always sum to $100 \%$ because of rounding errors.

${ }^{*}$ Post-hoc test, $\mathrm{p}<0.05$, normal group vs mild OSA group.

$\uparrow$ Post-hoc test, $p<0.05$, normal group vs moderate to severe OSA group.

$\$$ Post-hoc test, $\mathrm{p}<0.05$, mild OSA group vs moderate to severe OSA group.

$\mathrm{AHI}$, apnoea-hypopnoea index; $\mathrm{Spo}_{2}$, oxygen haemoglobin saturation. 
Figure 1 Systolic (A) and diastolic (B) blood pressure (BP) during wakefulness and sleep in different groups. Values are mean $(95 \% \mathrm{Cl})$. *Post-hoc test, $\mathrm{p}<0.05$, normal group vs mild obstructive sleep apnoea (OSA) group; †post-hoc test $\mathrm{p}<0.05$, normal group vs moderate to severe OSA group; tpost-hoc test, $\mathrm{p}<0.05$, mild OSA group vs moderate to severe OSA group.

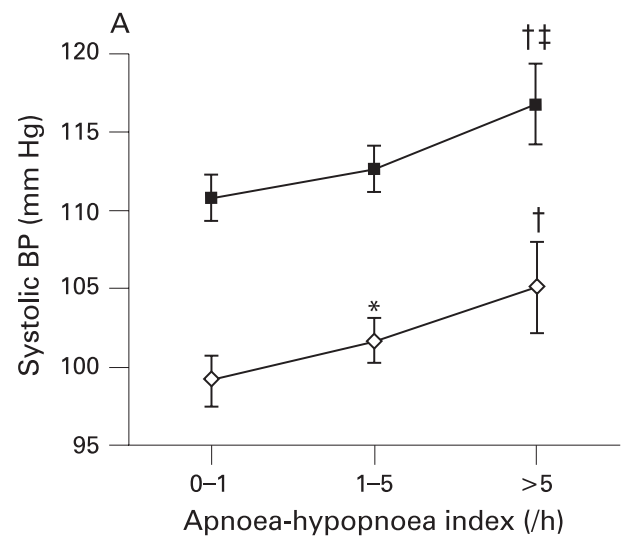

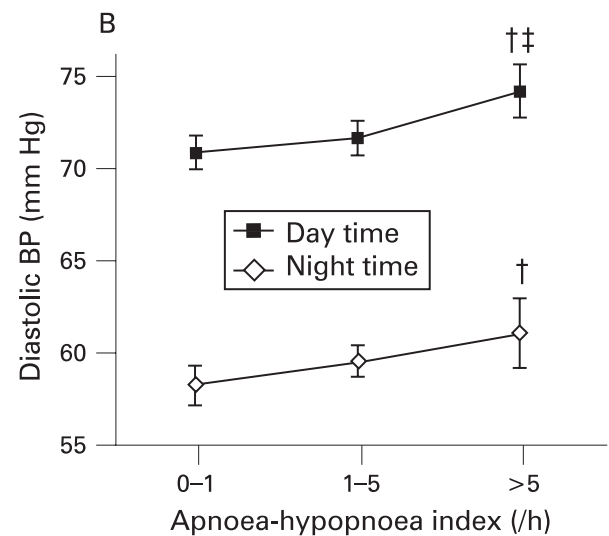

Multiple linear regression analyses were performed to assess the relationship between polysomnographic variables and BP measures, while controlling for possible confounders.

Logistic regression analyses were performed to calculate the odds ratio (OR) of different forms of hypertension (systolic or diastolic hypertension during wake or sleep) for different severities of OSA compared with normal controls, while adjusting for possible confounding factors. All analyses were performed using the SPSS (Statistical Package for the Social Sciences) for Windows V.13.0.

\section{RESULTS}

\section{Subject characteristics}

A total of 619 subjects were admitted for PSG but only 466 completed the BP recording. This was because there were four subjects admitted for PSG every working day but our unit only had three BP recording machines. Three out of the four subjects were thus randomly selected for recording and none of them refused.

Of the 466 subjects who underwent both PSG and ABP recording, 118 children were classified as primary snorers and

Table 2 Group comparison of blood pressure measures

\begin{tabular}{|c|c|c|c|c|c|}
\hline & $\begin{array}{l}\text { Entire sample } \\
(n=306)\end{array}$ & $\begin{array}{l}A H I \leqslant 1 \\
(n=127)\end{array}$ & $\begin{array}{l}1<\text { AHI } \leqslant 5 \\
(n=133)\end{array}$ & $\begin{array}{l}A H I>5 \\
(n=46)\end{array}$ & $\begin{array}{l}\text { p Value } \\
\text { (trend) }\end{array}$ \\
\hline \multicolumn{6}{|l|}{ During wakefulness } \\
\hline Systolic BP $(\mathrm{mm} \mathrm{Hg}) \dagger+$ & $112.5(8.4)$ & $110.8(8.3)$ & $112.6(8.0)$ & $116.7(8.4)$ & $<0.001$ \\
\hline Diastolic BP $(\mathrm{mm} \mathrm{Hg}) \dagger+$ & $71.6(5.1)$ & $70.8(5.0)$ & $71.6(4.9)$ & $74.1(4.8)$ & $<0.001$ \\
\hline Mean arterial pressure $(\mathrm{mm} \mathrm{Hg}) \uparrow \dagger$ & $85.1(5.4)$ & $84.1(5.2)$ & $85.0(5.2)$ & $88.0(5.6)$ & $<0.001$ \\
\hline Systolic BP z score $†$ † & $-0.09(1.06)$ & $-0.28(1.05)$ & $-0.10(1.00)$ & $0.47(1.06)$ & $<0.001$ \\
\hline Diastolic BP z score $\dagger$ † & $-0.06(0.84)$ & $-0.20(0.82)$ & $-0.07(0.82)$ & $0.34(0.84)$ & $<0.001$ \\
\hline Mean arterial pressure z score $\dagger+$ & $0.07(0.81)$ & $-0.06(0.76)$ & $0.05(0.77)$ & $0.53(0.90)$ & $<0.001$ \\
\hline Elevated systolic BP (n (\%))††§ & $30(9.8)$ & $9(7.1)$ & $10(7.5)$ & $11(23.9)$ & 0.007 \\
\hline Systolic hypertension (n (\%))§ & $17(5.6)$ & $5(3.9)$ & $7(5.3)$ & $5(10.9)$ & 0.11 \\
\hline Elevated diastolic BP (n (\%))§ & $20(6.5)$ & $6(4.7)$ & $10(7.5)$ & $4(8.7)$ & 0.28 \\
\hline Diastolic hypertension (n (\%))§ & $11(3.6)$ & $2(1.6)$ & $6(4.5)$ & $3(6.5)$ & 0.09 \\
\hline \multicolumn{6}{|l|}{ During sleep } \\
\hline Systolic BP $\left(\mathrm{mm} \mathrm{Hg}^{*}\right)^{*}$ & $101.2(9.0)$ & $99.1(9.1)$ & $101.7(8.0)$ & $105.1(9.8)$ & $<0.001$ \\
\hline Diastolic BP $(\mathrm{mm} \mathrm{Hg}) \dagger$ & $59.3(5.7)$ & $58.3(6.0)$ & $59.5(4.9)$ & $61.1(6.4)$ & 0.003 \\
\hline Mean arterial pressure $(\mathrm{mm} \mathrm{Hg})^{*} \dagger$ & $74.5(5.7)$ & $73.2(6.0)$ & $75.0(4.9)$ & $76.7(6.4)$ & $<0.001$ \\
\hline Systolic BP z score $\dagger$ & $0.38(1.05)$ & $0.15(1.09)$ & $0.44(0.92)$ & $0.84(1.10)$ & $<0.001$ \\
\hline Diastolic BP z score $†$ & $0.66(0.93)$ & $0.51(0.98)$ & $0.71(0.82)$ & $0.93(1.04)$ & 0.006 \\
\hline Mean arterial pressure $z$ score ${ }^{*} \dagger$ & $0.78(0.82)$ & $0.59(0.87)$ & $0.85(0.69)$ & $1.08(0.91)$ & $<0.001$ \\
\hline Elevated systolic BP (n (\%))†§ & $54(17.6)$ & $18(14.2)$ & $22(16.5)$ & $14(30.4)$ & 0.03 \\
\hline Systolic hypertension $(\mathrm{n}(\%)) \dagger+\S$ & $37(12.1)$ & $13(10.2)$ & $11(8.3)$ & $13(28.3)$ & 0.02 \\
\hline Elevated diastolic BP (n (\%))§ & $69(22.5)$ & $27(21.3)$ & $27(20.3)$ & $15(32.6)$ & 0.22 \\
\hline Diastolic hypertension $(\mathrm{n}(\%)) \dagger \uparrow \S$ & $48(15.7)$ & $15(11.8)$ & $18(13.5)$ & $15(32.6)$ & 0.005 \\
\hline \multicolumn{6}{|l|}{ Nocturnal dipping } \\
\hline Systolic BP dipping (\%) & $10.0(5.6)$ & $10.5(5.4)$ & $9.6(5.7)$ & $9.9(5.8)$ & 0.34 \\
\hline Diastolic BP dipping (\%) & $17.2(6.7)$ & $17.5(6.9)$ & $16.7(6.4)$ & $17.5(7.1)$ & 0.68 \\
\hline Mean arterial pressure dipping (\%) & $12.3(5.4)$ & $13.0(5.2)$ & $11.6(5.5)$ & $12.8(5.2)$ & 0.40 \\
\hline Systolic BP non-dipper (n (\%)) & $146(47.7)$ & $59(46.5)$ & $66(49.6)$ & $21(45.7)$ & 0.92 \\
\hline Diastolic BP non-dipper (n (\%)) & $41(13.4)$ & $14(11.0)$ & $19(14.3)$ & $8(17.4)$ & 0.25 \\
\hline Mean arterial pressure non-dipper (n (\%)) & $97(31.7)$ & $36(28.3)$ & $49(36.8)$ & $12(26.1)$ & 0.77 \\
\hline
\end{tabular}

Mean (SD) and number (\%) are presented for parametric and categorical data, respectively.

*Post-hoc test, $\mathrm{p}<0.05$, normal group vs mild OSA group.

$\uparrow$ Post-hoc test, $p<0.05$, normal group vs moderate to severe OSA group.

$\$$ Post-hoc test, $p<0.05$, mild OSA group vs moderate to severe OSA group.

$\S p<0.016$ was considered to be significant in multiple pairwise comparison

AHI, apnoea-hypopnoea index; BP, blood pressure; OSA, obstructive sleep apnoea. 
Table 3 Multiple linear regression analyses showing the association between polysomnographic parameters and daytime blood pressure parameters before and after adjustment for possible confounders

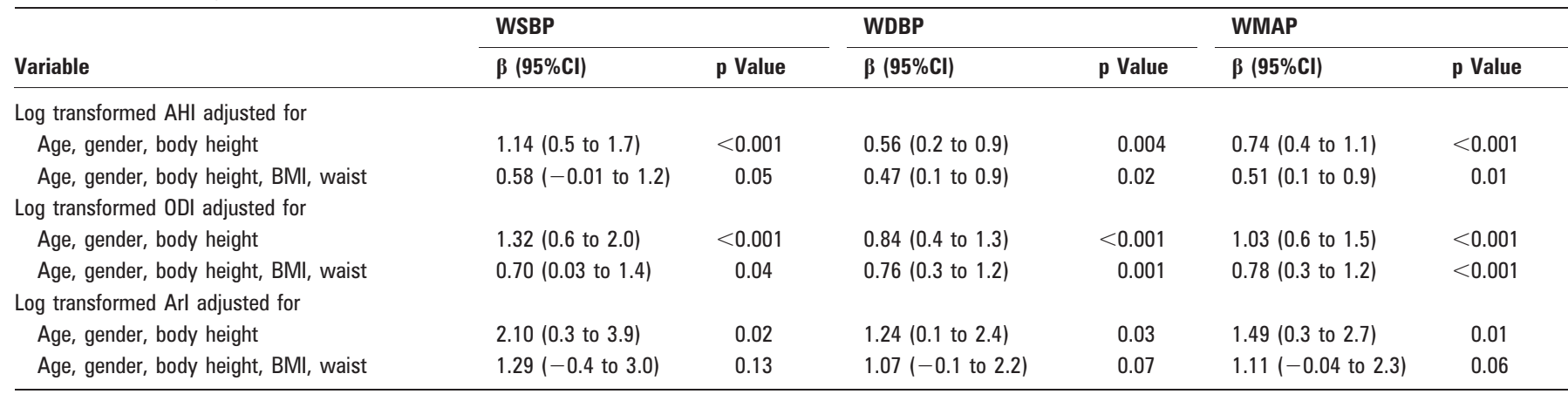

$\mathrm{AHI}$, apnoea-hypopnoea index; Arl, arousal index; $\beta$ (SE), beta coefficient (standard error); BMI, body mass index; ODI, oxygen desaturation index; waist, waist circumference WDBP, wake diastolic blood pressure; WMAP, wake mean arterial pressure; WSBP, wake systolic blood pressure.

were excluded. Forty-two subjects were also excluded as their $\mathrm{BP}$ recording did not satisfy our minimal criteria for success. As a result, 306 subjects were included in the final analysis. The demographic, anthropometric and PSG characteristics are presented in table 1. Compared with normal controls and subjects with a lower AHI, the group with AHI $>5$ included a larger proportion of boys with higher BMI z score. As expected, subjects with higher AHI values also had significantly higher oxygen desaturation index, arousal index and lower oxygen haemoglobin saturation nadir.

\section{Characteristics of ambulatory blood pressure measurement}

There was no significant difference in the characteristics of $A B P$ recording between groups. The median (IOR) total recording time for all subjects was 16.5 (15.0-22.9). The median (IOR) number of BP readings during wakefulness and sleep were 13 (10-20) and 9 (8-9) respectively (table 1 of the online supplement).

Selection of seven BP readings during sleep was based on the recommendation of the European Society of Hypertension. ${ }^{18}$ Many of our subjects did not comply with the recommendation for daytime BP recording because of schooling and other social commitments. The selection of seven daytime readings was arbitrary and we had analysed our data to verify that this selection was valid and representative. Subjects with at least 14 successful daytime BP readings were selected, and comparisons were made between the average daytime BP levels of the entire recording and the average levels of the first seven readings. There were 138 subjects with at least 14 successful daytime readings and there were no significant differences between the overall $\mathrm{BP}$ averages and the averages of the first seven readings (see table 2 of the online supplement). Based on these data, we believed that seven successful daytime BP recordings were representative.

\section{Blood pressure during wakefulness}

Post hoc pairwise comparison showed that the moderate to severe OSA group had significantly higher wake systolic (WSBP), diastolic (WDBP) and mean arterial BP (WMAP) than both the normal control and the mild OSA group, while no significant differences were found between the normal and the mild group (fig 1). The same results were obtained after converting the wake BP parameters into $z$ scores (adjusting for gender and height). Furthermore, the moderate to severe group included a significantly greater proportion of subjects having elevated WSBP (table 2). In the logistic regression analyses adjusted for only age and gender, subjects with moderate to severe OSA had a higher odds ratio for elevated WSBP compared with normal controls (OR 3.94 (95\% confidence interval (95\% CI) 1.49 to 10.43)). However, once adjusted for BMI and waist circumference, the significant difference in the risk of elevated WSBP was no longer demonstrated (OR 2.12 (95\% CI 0.73 to 6.13)) (see table 3 of the online supplement).

Multiple linear regression analysis showed that the associations between polysomnographic parameters and WSBP or WMAP were partially explained by BMI and waist circumference, whereas the associations with WDBP were only slightly confounded by BMI and waist circumference. Oxygen desaturation index had the strongest association with wake BP measures (table 3).

Table 4 Multiple linear regression analyses showing the association between polysomnographic parameters and nocturnal blood pressure parameters before and after adjustment for possible confounders

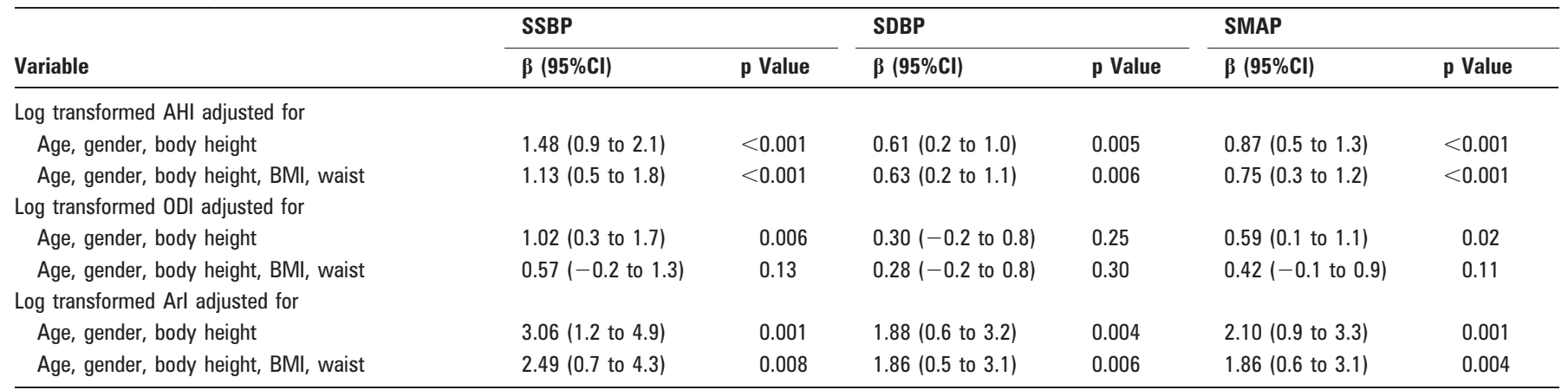

AHI, apnoea-hypopnoea index; Arl, arousal index; $\beta$ (SE), beta coefficient (standard error); BMI, body mass index; ODI, oxygen desaturation index; SDBP, sleep diastolic blood pressure; SMAP, sleep mean arterial pressure; SSBP, sleep systolic blood pressure; waist, waist circumference. 
Table 5 Blood pressure measurements of non-overweight subjects

\begin{tabular}{|c|c|c|c|c|c|}
\hline & $\begin{array}{l}\text { Entire sample } \\
(\mathrm{n}=204)\end{array}$ & $\begin{array}{l}\text { AHI } \leqslant 1 \\
(n=103)\end{array}$ & $\begin{array}{l}1<\text { AHI } \leqslant \mathbf{5} \\
(\mathrm{n}=\mathbf{8 5})\end{array}$ & $\begin{array}{l}A H I>5 \\
(n=16)\end{array}$ & $\begin{array}{l}\text { p Value } \\
\text { (trend) }\end{array}$ \\
\hline Age $(y)$ & $10.4(1.7)$ & $10.4(1.8)$ & $10.4(1.6)$ & $10.3(1.7)$ & 0.91 \\
\hline Body mass index $\left(\mathrm{kg} / \mathrm{m}^{2}\right)$ & $16.5(1.9)$ & $16.4(2.0)$ & $16.6(1.9)$ & $16.6(1.6)$ & 0.53 \\
\hline \multicolumn{6}{|l|}{ During wakefulness } \\
\hline Systolic BP (mm Hg) & $109.8(7.3)$ & $109.0(7.5)$ & $110.5(7.2)$ & $111.1(6.4)$ & 0.11 \\
\hline Diastolic BP $(\mathrm{mm} \mathrm{Hg}) \dagger$ & $70.9(4.8)$ & $70.2(4.8)$ & $71.2(4.6)$ & $73.6(4.9)$ & 0.011 \\
\hline Mean arterial pressure $(\mathrm{mm} \mathrm{Hg})$ & $83.8(4.9)$ & $83.2(5.0)$ & $84.1(4.7)$ & $85.7(4.5)$ & 0.047 \\
\hline Systolic BP z score & $-0.41(0.94)$ & $-0.52(0.95)$ & $-0.32(0.95)$ & $-0.26(0.81)$ & 0.13 \\
\hline Diastolic BP z score $\dagger$ & $-0.18(0.78)$ & $-0.28(0.78)$ & $-0.15(0.75)$ & $0.26(0.82)$ & 0.014 \\
\hline Mean arterial pressure z score & $-0.11(0.71)$ & $-0.18(0.73)$ & $-0.07(0.70)$ & $0.15(0.66)$ & 0.064 \\
\hline \multicolumn{6}{|l|}{ During sleep } \\
\hline Systolic BP (mm Hg) & $98.7(8.3)$ & $97.3(8.0)$ & $100.0(7.9)$ & $100.9(10.4)$ & 0.016 \\
\hline Diastolic BP (mm Hg) & $58.8(5.4)$ & $57.9(5.5)$ & $59.6(4.7)$ & $60.5(6.6)$ & 0.015 \\
\hline Mean arterial pressure $(\mathrm{mm} \mathrm{Hg})^{*}$ & $73.4(5.4)$ & $72.3(5.4)$ & $74.4(4.9)$ & $74.6(6.3)$ & 0.007 \\
\hline Systolic BP z score* & $0.12(1.00)$ & $-0.07(0.97)$ & $0.30(0.96)$ & $0.40(1.25)$ & 0.008 \\
\hline Diastolic BP z score & $0.60(0.90)$ & $0.45(0.92)$ & $0.72(0.80)$ & $0.85(1.13)$ & 0.019 \\
\hline Mean arterial pressure $z$ score ${ }^{*}$ & $0.63(0.77)$ & $0.47(0.78)$ & $0.78(0.70)$ & $0.80(0.93)$ & 0.006 \\
\hline
\end{tabular}

Mean (SD) are presented.

*Post-hoc test, $p<0.05$, normal group vs mild OSA group.

$\uparrow$ Post-hoc test, $p<0.05$, normal group vs moderate to severe OSA group.

$\mathrm{AHI}$, apnoea-hypopnoea index; BP, blood pressure; OSA, obstructive sleep apnoea.

\section{Blood pressure during sleep}

One way ANOVA showed that subjects with moderate to severe OSA had significantly higher sleep systolic BP (SSBP), sleep diastolic BP (SDBP) and sleep mean arterial BP (SMAP) compared with controls (fig 1). This finding remained the same after converting the sleep BP parameters into z scores (adjusting for gender and height). Furthermore, compared with controls and those with lower AHI values, subjects with AHI $>5$ included a significantly greater proportion of subjects with sleep systolic and diastolic hypertension (table 2).

In the logistic regression analyses adjusted for age and gender, subjects with AHI $>5$ had approximately 5 times and 3.5 times higher risk for sleep systolic (OR 5.0 (95\% CI 2.0 to 12.7)) and diastolic (OR 3.5 (95\% CI 1.5 to 8.1)) hypertension, respectively, compared with healthy controls. The ORs were reduced to 3.9 (95\% CI 1.4 to 10.5 ) and 3.3 (95\% CI 1.4 to 8.1), respectively, after adding BMI and waist circumference into the model. In contrast, for the group with AHI between 1 and 5, no significant increased risk for hypertension or elevated BP was found compared with the healthy controls (see table 3 of the online supplement).

Multiple linear regression analyses showed that the association between polysomnographic parameters and SSBP or SMAP were partially explained by BMI and waist circumference whereas the associations between SDBP and polysomnographic parameters were not significantly affected by BMI and waist circumference. AHI had the strongest association with elevated nocturnal BP (table 4).

\section{Nocturnal BP dipping}

No significant differences in nocturnal BP dipping and proportion of non-dippers were identified between the three groups (table 2).

\section{Subgroup analysis of non-overweight children}

To further verify that the effect of OSA on BP elevation was independent of obesity, subgroup analyses were performed after excluding overweight subjects (BMI greater than the 85th percentile specific for age and gender). There were no significant differences in BMI, BMI z score or waist circumference between the different groups (table 5).

Across the groups, significant increasing trends were identified for all BP measurements during sleep. Significant differences in mean arterial pressure, systolic BP z score and mean arterial pressure $z$ score were found between controls and those with mild OSA but not between the moderate to severe group and the controls. The latter might be because of the small number of subjects in the moderate to severe group. For daytime parameters, diastolic BP, mean arterial pressure and diastolic BP $z$ score had significant increasing trends across groups. Significant differences in diastolic BP and its $z$ score were identified between the moderate to severe group and controls.

\section{DISCUSSION}

Our study has provided robust evidence that children with OSA had significantly higher BP than normal healthy children during both sleep and wakefulness. BP levels increased with the severity of OSA, and children with moderate to severe disease (AHI >5) were at significantly higher risk for nocturnal hypertension. The effect of childhood OSA on BP levels was independent of the effect of obesity. These findings have important clinical implications as the current literature suggests elevated childhood BP mediates adulthood hypertension and metabolic syndrome. ${ }^{21}$ In addition, elevated BP during childhood is associated with increased carotid artery intima-media thickness, which precedes the development of atherosclerosis. ${ }^{22}$

Both daytime systolic and diastolic BP were found to be significantly higher in children with moderate to severe OSA compared with those with mild disease and healthy controls. Although statistically insignificant, there was a trend in the same direction for both daytime systolic and diastolic hypertension. This is a novel and important finding as childhood BP could determine future adverse cardiovascular problems. Two previous paediatric studies using ambulatory BP recording failed to show results similar to ours. Amin and colleagues ${ }^{5}$ evaluated 60 snoring children and found lower daytime diastolic BP in children with more severe OSA (AHI >5). The investigators suggested that such a finding was a reflection of abnormal elastic recoil of blood vessels during diastole. Casual BP instead 
of ABP normal references were used in Amin's study and that could have given rise to inappropriate BP interpretation. ${ }^{23} \mathrm{~A}$ more recent study also failed to provide support for daytime BP elevation in children with OSA. ${ }^{9}$ The study included only 17 cases with severe OSA and did not have a normal control group for comparison.

Our current study documented higher nocturnal systolic and diastolic BP in children with moderate to severe OSA compared with healthy subjects. In addition, the same group of children also had a significantly higher prevalence of nocturnal systolic and diastolic hypertension. Children with AHI $>5$ had an odds ratio of 3.9 (95\% CI 1.4 to 10.5$)$ and 3.3 (1.4 to 8.1) for nocturnal systolic and diastolic hypertension, respectively. Another important finding is demonstration of significant differences in nocturnal systolic and mean arterial BP between children with mild OSA and normal controls. Such differences in BP measures were not seen in the daytime recording. This may suggest nocturnal $\mathrm{BP}$ in children is more liable to elevation as a result of OSA compared with wake BP. A similar finding was also reported in adults where nocturnal hypertension was the more frequent pattern of hypertension seen in patients with newly diagnosed OSA. ${ }^{24}$ This emphasises the importance of nocturnal rather than daytime BP measurement in children suspected of having sleep apnoea.

The effect size of OSA severity on BP levels was small, which could be explained by the community based recruitment of our subjects. A recent paediatric study ${ }^{25}$ found that a fully adjusted beta coefficient of log transformed AHI on nocturnal systolic BP of 0.5 was associated with significant left ventricular abnormalities, which are important markers of future cardiovascular adverse events. ${ }^{26}{ }^{27}$ Therefore, our findings of elevated BP, although small, would still have important and relevant long term health implications.

Non-dipping BP has been associated with hypertensive target organ damage. ${ }^{28}$ We failed to find any significant differences in non-dipping BP profile between the three groups. In contrast, Amin and colleagues ${ }^{5}$ documented a significantly greater proportion of subjects with moderate to severe OSA as having non-dipping BP. This discrepancy could be explained by the more severe OSA status and a greater proportion of obese children being included in their study.

Repetitive arousals and recurrent hypoxic events caused by intermittent airway occlusion are proposed mechanisms linking OSA and systemic hypertension. ${ }^{29}$ Both mechanisms are thought to mediate sympathetic activation that may overspill into the day leading to hypertension during the day. Our findings showed that of these two mechanisms, nocturnal hypoxaemia was more closely associated with daytime BP elevation while recurrent arousals, resulting in sleep fragmentation, was more closely related to the increase in nocturnal BP. Our results were also consistent with a previous adult study which suggested that nocturnal hypoxaemia was associated with sympathetic activation that spilled over at least into the first hour after waking up and caused daytime hypertension. ${ }^{30} \mathrm{~A}$ canine model of OSA documented sustained daytime hypertension after dogs were exposed to recurrent apnoeas. However, such BP changes were not seen when the animals were exposed to recurrent arousals only. ${ }^{31}$

One of the limitations of our study was the low compliance for a full $24 \mathrm{~h}$ BP recording. Many of our subjects were unwilling to comply with monitoring over a $24 \mathrm{~h}$ period because of school or other social commitments, a problem commonly encountered in paediatric research. Nonetheless, all of our sleep $\mathrm{BP}$ measurements satisfied the criteria needed for adequate $\mathrm{ABP}$ monitoring, ${ }^{16}$ and the seven daytime readings were proven to be representative. Ambulatory normal data from a German cohort was used in our study, as local reference values were not available. As a result, the blood pressure $z$ score and the 90th and 95 th percentiles may be subjected to racial errors and should be interpreted with caution. Our study, however, has major advantages over other published paediatric series. Firstly, and most importantly, our study included the largest sample size to date reported in the literature. In addition, we also included a normal healthy control group for comparison. Primary snorers used by other studies as controls were inappropriate as a previous publication documented higher BP in primary snorers than in non-snorers. ${ }^{32}$ We avoided sampling bias by recruiting subjects from the community instead of from hospital attendants. This would have minimised bias or other unknown factors that influence referral to hospital clinics. Lastly, we used ambulatory measurements rather than casual readings to provide a more reliable evaluation of BP changes. ${ }^{11}$

\section{CONCLUSION}

BP levels during both wakefulness and sleep increased with the severity of childhood OSA. Children with more severe disease (AHI >5) were at increased risk of nocturnal hypertension. These findings are clinically relevant and important as high BP is a well established risk factor for future adverse cardiovascular events, and children with OSA are thus predisposed to such a risk. Therefore, children with symptoms suggestive of OSA should be investigated early, and future studies should examine the effect of therapy for OSA on BP changes.

Acknowledgements: We are grateful for the logistic support of all the staff of the Sleep Assessment Unit at Shatin Hospital, and the cooperation and participation of all the schools, children and their parents.

Funding: This study was supported by grant (CUHK4161/02M) from the Research Grants Council of the Hong Kong Special Administrative Region, China.

Competing interests: None.

Ethics approval: Ethics approval was obtained

\section{REFERENCES}

1. Nieto FJ, Young TB, Lind BK, et al. Association of sleep-disordered breathing, sleep apnea and hypertension in a large community-based study: Sleep Heart Health Study. JAMA 2000;283:1829-36.

2. Peppard PE, Young T, Palta M, Skatrud J. A prospective study of the association between sleep-disordered breathing and hypertension. N Engl J Med 2000;342:1378-84.

3. Shahar E, Whitney CW, Redline S, et al. Sleep-disordered breathing and cardiovascular disease: cross-sectional results of the Sleep Heart Health Study. Am J Respir Crit Care Med 2001;163:19-25.

4. Marcus CL, Greene MG, Carroll JL. Blood pressure in children with obstructive sleep apnea. Am J Respir Crit Care Med 1998;157:1098-103.

5. Amin RS, Carroll JL, Jeffries JL, et al. Twenty-four-hour ambulatory blood pressure in children with sleep-disordered breathing. Am J Respir Crit Care Med 2004;169:950-6.

6. Guilleminault C, Khramsov A, Stoohs RA, et al. Abnormal blood pressure in prepubertal children with sleep-disordered breathing. Pediatr Res 2004;55:76-84.

7. Kohyama J, Ohinata JS, Hasegawa T. Blood pressure in sleep disordered breathing Arch Dis Child 2003;88:139-42.

8. Enright PL, Goodwin JL, Sherrill DL, et al. Blood pressure elevation associated with sleep-related breathing disorder in a community sample of white and hispanic children. Arch Pediatr Adolesc Med 2003;157:901-4.

9. Leung LC, Ng DK, Lau MW, et al. Twenty-four-hour ambulatory BP in snoring children with obstructive sleep apnea syndrome. Chest 2006;130:1009-17.

10. Zintzaras E, Kaditis AG. Sleep-disordered breathing and blood pressure in childrenA meta-analysis. Arch Pediatr Adolesc Med 2007;161:172-8.

11. Verdecchia P, Porcellati C, Schillaci G, et al. Ambulatory blood pressure: an independent predictor of prognosis in essential hypertension. Hypertension 1994;24:793-801.

12. Li AM, Cheung A, Chan D, et al. Validation of a questionnaire instrument for prediction of obstructive sleep apnea in Hong Kong Chinese children. Pediatr Pulmonol 2006;41:1153-60.

13. Leung SS, Cole TJ, Tse LY, et al. Body mass index reference curves for Chinese children. Ann Hum Biol 1998;25:169-74. 
14. Wing YK, Hui SH, Pak WM, et al. A controlled study of sleep related disordered breathing in obese children. Arch Dis Child 2003;88:1043-7.

15. American Thoracic Society. Cardiorespiratory sleep studies in children: establishment of normative data and polysomnographic predictors of morbidity. Am J Respir Crit Care Med 1999;160:1381-7.

16. O'Brien E, Asmar R, Beilin L, et al. European Society of Hypertension Working group on Blood Pressure Monitoring. European Society of Hypertension recommendations for conventional, ambulatory and home blood pressure measurement. J Hypertens 2003;21:821-48.

17. Wuhl E, Witte K, Soergel M, et al. German Working Group on Pediatric Hypertension. Distribution of 24-hour ambulatory blood pressure in children: normalized reference values and role of body dimensions. J Hypertens 2002;20:1995-2007.

18. Cole TJ, Green PJ. Smoothing reference centile curve: the LMS method and penalized likelihood. Stat Med 1992;11:1305-19.

19. Morgan H, Khan I, Hashmi A, et al. Ambulatory blood pressure monitoring after renal transplantation in children. Pediatr Nephrol 2001;16:843-7.

20. Soergel M, Kirschstein M, Busch C, et al. Oscillometric twenty-four-hour ambulatory blood pressure values in healthy children and adolescents: a multicenter trial including 1141 subjects. J Pediatr 1997;130:178-84.

21. Sun SS, Grave GD, Siervogel RM, et al. Systolic blood pressure in children predicts hypertension and metabolic syndrome later in life. Pediatrics 2007;119:237-46.

22. Li S, Chen W, Srinivasan SR, et al. Childhood cardiovascular risk factors and carotid vascular changes in adulthood: the Bogalusa Heart Study. JAMA 2003;290:2271-6.
23. Sorof JMM, Poffenbarger T, Franco $\mathrm{K}$, et al. Evaluation of white coat hypertension in children: importance of the definitions of normal ambulatory blood pressure and the severity of casual hypertension. Am J Hypertens 2001;14:855-60.

24. Baguet JP, Hammer L, Lévy P, et al. Night-time and diastolic hypertension are common and underestimated conditions in newly diagnosed apnoeic patients. $J$ Hypertens 2005;23:521-7.

25. Amin R, Somers VK, McConnell K, et al. Activity-adjusted 24-hour ambulatory blood pressure and cardiac remodeling in children with sleep disordered breathing. Hypertension 2008;51:84-91.

26. Vakili BA, Okin PM, Devereux RB. Prognostic implications of left ventricular hypertrophy. Am Heart J 2001;141:334-41.

27. Verdecchia $\mathbf{P}$, Schillaci G, Borgioni $\mathrm{C}$, et al. Adverse prognostic significance of concentric remodeling of the left ventricle in hypertensive patients with normal left ventricular mass. J Am Coll Cardiol 1995;25:871-8.

28. Birkenhager $\mathbf{A M}$, van den Meiracker $\mathrm{AH}$. Causes and consequences of a nondipping blood pressure profile. Neth J Med 2007;65:127-131.

29. Ringler J, Basner RC, Shannon R, et al. Hypoxemia alone does not explain blood pressure elevations after obstructive apnea. J Appl Physiol 1990;69:2143-8.

30. Peled N, Greenberg A, Pillar G, et al. Contributions of hypoxia and respiratory disturbance index to sympathetic activation and blood pressure in obstructive sleep apnea syndrome. Am J Hypertens 1998;11:1284-9.

31. Brooks D, Horner RL, Kozar LF, et al. Obstructive sleep apnea as a cause of systemic hypertension. Evidence from a canine model. J Clin Invest 1997:99:106-9.

32. Kwok KL, Ng DK, Cheung YF. BP and arterial distensibility in children with primary snoring. Chest 2003;123:1561-6. 